\title{
Eficácia do Implante Valvar Aórtico Transcateter (TAVI) na estenose valvar aórtica
}

\author{
Efficacy of Transcatheter Aortic Valve Implant (TAVI) in aortic valve stenosis
}

Eficacia del implante de válvula aórtica transcatéter (TAVI) en la estenosis de la válvula aórtica

Mariana Por-Deus Vilela ${ }^{1 *}$, Jasmin Dezotti Lovisi², Júlia Aguiar Mendes², Letícia Lourenço Botelho ${ }^{3}$, Luana Miguez Albuquerque Silva ${ }^{4}$, Luiz Valério Costa Vasconcelos ${ }^{5}$, Mariana Calheiros Poloni ${ }^{6}$, Paula Meira Ferraz ${ }^{7}$, Raíssa Frederico Giacomin ${ }^{8}$, Humberto de Freitas Boy ${ }^{9}$.

\section{RESUMO}

Objetivo: Esclarecer a eficácia do Implante Valvar Aórtico Transcateter (TAVI) no tratamento da estenose valvar aórtica revisando suas evidências clínicas, comparando com o tratamento cirúrgico convencional e evidenciando suas limitações, complicações e custo efetividade a partir da análise da produção científica atual sobre o tema. Revisão bibliográfica: A Estenose Aórtica (EA) é uma doença comum do sistema cardiovascular e que pode levar à deterioração progressiva do ventrículo esquerdo. Em muitos casos, a intervenção cirúrgica é necessária a fim de garantir a sobrevida do paciente. Dentre as técnicas disponíveis, o TAVI, quando comparado à cirurgia convencional, garante áreas valvares maiores e com melhores desempenhos hemodinâmicos, reduz o tempo cirúrgico e de recuperação pós-operatória e, ainda, mostrouse superior em relação à ocorrência de algumas complicações. Dessa forma, o TAVI é tido como técnica padrão-ouro para pacientes de alto risco e uma opção para médio e baixo risco. Considerações finais: $O$ TAVI apresenta benefícios no tratamento da EA, principalmente em pacientes graves onde a cirurgia convencional é contraindicada. Entretanto, o custo, a falta de conhecimento médico sobre a técnica e de estrutura hospitalar adequada podem ser fatores limitantes no nosso país.

Palavras-chave: Eficácia, Implante de prótese de valva cardíaca, Estenose da valva aórtica.

\footnotetext{
ABSTRACT

${ }^{1}$ Instituto Master de Ensino Presidente Antonio Carlos (IMEPAC), Araguari - MG.

*E-mail: marianavilela65@gmail.com

2 Faculdade de Medicina Souza Marques (FTESM), Rio de Janeiro - RJ.

${ }^{3}$ Centro Universitário de Valença (UNIFAA), Valença - RJ.

${ }^{4}$ Universidade Vale do Rio Doce (UNIVALE), Governador Valadares - MG.

${ }^{5}$ Universidade de Fortaleza (Unifor), Fortaleza - CE.

${ }^{6}$ Universidade Estadual do Oeste do Paraná (UNIOESTE), Cascavel - PR.

${ }^{7}$ Faculdade de Ciências Gerenciais (FACIG), Manhuaçu - MG.

${ }^{8}$ Centro Universitário do Espírito Santo (UNESC), Colatina - ES.

${ }^{9}$ Centro Universitário de Caratinga (UNEC), Caratinga - MG.
}

Objective: To make clear the efficacy of transcatheter aortic valve implantation (TAVI) in the treatment of aortic valve stenosis by reviewing the clinical evidence, comparing it with conventional surgical treatment, and highlighting the limits, complications, and value for money from an analysis of the current scientific production on the subject. Bibliographic review: Aortic stenosis (AS) is a common disease of the cardiovascular system that can lead to progressive deterioration of the left ventricle. In many cases, surgical intervention is necessary in an effort to guarantee the patient's survival. Among the available techniques, TAVI, when compared to conventional surgery, ensures larger valve areas with better hemodynamic performance, reduces the surgical time and postoperative recovery, and also proved to be superior in relation to the incidence of some 
complications. Therefore, TAVI is considered the gold standard technique for high-risk patients and an option for medium and low risk. Final considerations: TAVI has benefits in the treatment of AS, especially in severe patients in which conventional surgery is contraindicated. However, the cost, lack of medical knowledge about the technique and adequate hospital structure may be limiting factors in our country.

Key words: Efficacy, Heart valve proshtesis implantation, Aortic valve stenosis.

\section{RESUMEN}

Objetivo: Aclarar la efectividad del Implante Valvular Aórtico Transcatéter (TAVI) en el tratamiento de la estenosis valvular aórtica revisando su evidencia clínica, comparándola con el tratamiento quirúrgico convencional y destacando sus limitaciones, complicaciones y coste-efectividad a partir del análisis de la producción científica actual sobre el tema. Revisión bibliográfica: La estenosis aórtica (EA) es una enfermedad común del sistema cardiovascular y puede conducir a un deterioro progresivo del ventrículo izquierdo. En muchos casos, la intervención quirúrgica es necesaria para asegurar la supervivencia del paciente. Entre las técnicas disponibles, la TAVI, en comparación con la cirugía convencional, garantiza áreas valvulares más grandes con mejor rendimiento hemodinámico, reduce el tiempo quirúrgico y la recuperación postoperatoria, y también demostró ser superior en relación con la aparición de algunas complicaciones. Así, la TAVI se considera la técnica de referencia para los pacientes de alto riesgo y una opción para los de riesgo medio y bajo. Consideraciones finales: EI TAVI tiene ventajas en el tratamiento de la EA, especialmente en pacientes graves en los que la cirugía convencional está contraindicada. Sin embargo, el coste, la falta de conocimientos médicos sobre la técnica y la estructura hospitalaria adecuada pueden ser factores limitantes en nuestro país.

Palabras clave: Eficacia, Implantación de prótesis de válvulas cardíacas, Estenosis de la válvula aórtica.

\section{INTRODUÇÃO}

A Estenose Aórtica (EA) define-se pela restrição da abertura efetiva da valva aórtica, com diminuição do orifício de passagem da área valvar, alteração no fluxo de ejeção do sangue pelo Ventrículo Esquerdo (VE) e consequente formação de gradiente de pressão sistólica entre o VE e a artéria aorta. Sua fisiopatologia está relacionada ao obstáculo à ejeção do VE com aumento progressivo da pressão sistólica da câmara, inicialmente promovendo uma hipertrofia concêntrica. Após isso, ocorre a redução da complacência do VE com aumento na sua pressão diastólica final, que, por sua vez, favorece a hipertrofia excêntrica, culminando nos sintomas de insuficiência cardíaca (MORAIS LR, et al., 2021).

A principal etiologia da estenose aórtica é degenerativa senil devido à formação de placas de ateroma, que dificultam sua abertura. Com o crescente envelhecimento da população, essa se torna a valvopatia mais frequente. Outras etiologias são a febre reumática, principalmente em países em desenvolvimento, e a estenose aórtica congênita com valva aórtica bicúspide ou unicúspide que geralmente é encontrada em pacientes mais jovens. Trata-se de uma doença insidiosa, com o quadro clínico se baseando em um período assintomático longo, seguido de sintomas de descompensação cardíaca (queda na fração de ejeção, dilatação ventricular progressiva e surgimento de arritmia) que geram uma mortalidade elevada em poucos anos. Esses sintomas evoluem para a tríade clássica da estenose aórtica: dispneia, síncope e angina (FOLLADOR W, et al., 2018).

O diagnóstico é feito por meio do ecocardiograma, que fornece dados como a área valvar, aumentada nesta patologia (BORJA MM e OLVERA-ARREOLA SS, 2015). Já para o tratamento, tem-se como opção a cirurgia convencional de substituição da valva, entretanto, fatores como idade avançada, cirurgia cardíaca prévia, doença arterial coronariana, disfunção ventricular e hipertensão pulmonar são contraindicações a esse tipo de intervenção devido ao seu risco perioperatório muito elevado. Avalia-se que, para pelo menos um terço dos pacientes com estenose aórtica grave, a cirurgia convencional não seja aplicável (FOLLADOR, W, et al., 2018; AMARAL HLP, et al., 2021). 
Nesse contexto, o Implante Valvar Aórtico Transcateter (TAVI) foi desenvolvido e consiste na introdução de uma válvula aórtica artificial por meio de um catéter em uma grande veia periférica, ou por via transapical. Desta forma, a TAVI destaca-se como uma alternativa de procedimento menos invasivo, que oferece menor risco ao paciente, sendo mais utilizada para casos inoperáveis ou que têm alto risco para a substituição cirúrgica da válvula por esternotomia. Desfechos favoráveis como aumento de sobrevida, melhora da sintomatologia e, portanto, da qualidade de vida, reduzindo o número de novas hospitalizações foram amplamente documentados (PINTO BCM, et al., 2020; OTTO et al., 2017).

Atualmente, já foi evidenciado que a TAVI não possui inferioridade em relação à cirurgia de correção da estenose aórtica nos pacientes de risco cirúrgico elevado e intermediário e que é o procedimento de escolha em pacientes portadores de estenose aórtica grave e de risco cirúrgico extremo. No entanto, é um procedimento mais caro, mas que pode ser custo efetivo principalmente na EA grave (MANGIONE FM, et al., 2017; KUNTJORO et al., 2020).

Diante do exposto, o objetivo do trabalho foi esclarecer a eficácia da TAVI no tratamento da estenose valvar aórtica revisando suas evidências clínicas, comparando com o tratamento cirúrgico convencional e evidenciando suas limitações, complicações e custo efetividade a partir da análise da produção científica atual sobre o tema.

\section{REVISÃO BIBLIOGRÁFICA}

A Estenose Valvar Aórtica é uma das doenças do Sistema Cardiovascular mais comuns de países desenvolvidos. Esta patologia é resultado de um conjunto de processos responsáveis pelo espessamento dos folhetos da Valva Aórtica e sua calcificação. Sua evolução está associada à deterioração progressiva do ventrículo esquerdo, levando à hipertrofia compensatória deste (PIO G, et al., 2021).

No quadro de EA, ocorre a redução da luz da valva aórtica, associada a uma reduzida maleabilidade dos folhetos que a compõem. Essa redução pode ocorrer em decorrência de lesões, inflamações e calcificações, com espessamento do tecido, levando à obstrução parcial do fluxo de sangue do VE, em direção à artéria aorta (FOLLADOR W, et al., 2018).

A prevalência da EA aumenta com a idade, atingindo de 2,8\% a 4,6\% de indivíduos acima de 75 anos. A doença é mais observada em países como os Estados Unidos da América e países do continente europeu, em decorrência da maior população de indivíduos idosos, uma vez que a principal causa associada ao desenvolvimento desta patologia é a calcificação degenerativa da valva normal. Estudos mostram que a sobrevida é de, aproximadamente, $60 \%$ em 1 ano e de $32 \%$ em 5 anos. As intervenções cirúrgicas alteram esse prognóstico e, normalmente, apresentam baixa taxa de mortalidade, em torno de 4\% (KANWAR A, et al., 2018; PONTES J, et al., 2013).

No Brasil, a estenose aórtica tem duas etiologias mais comuns: a doença reumática, muito prevalente em todo o mundo principalmente em jovens, e a calcificação da valva aórtica, que aumenta sua prevalência com a idade (JOSEPH J, et al. 2017). Essa degeneração da valva aórtica se decorre por um processo ativo de remodelamento da valva decorrente de estresse mecânico, principalmente em portadores de valva bicúspide. O remodelamento ocorre com ação de fibroblastos, depósito de lipoproteínas como o LDL (lipoproteína de baixa densidade), e outras células imunológicas como macrófagos, linfócitos T e mastócitos. O mecanismo pelo qual ocorre a calcificação ainda é tema de muitos estudos (ZHENG KH, et al., 2019).

A estenose aórtica leva ao aumento da pós carga, exigindo mais do ventrículo esquerdo na sístole. Dessa forma, ocorre hipertrofia ventricular de forma compensatória à estenose aórtica. Inicialmente a hipertrofia do ventrículo esquerdo pode compensar, mas tende ao desequilíbrio, visto que a estenose valvar é progressiva e o ventrículo se torna disfuncional devido a hipertrofia, fibrose e até mesmo insuficiência cardíaca (LIU Z, et al., 2017; GRIMARD BH, et al., 2016).

A maior parte dos pacientes são assintomáticos apresentando apenas sopro mesossistólico, melhor auscultado em segundo espaço intercostal direito podendo irradiar para as artérias carótidas. As demais 
manifestações dependem do grau de severidade da estenose aórtica e comorbidades associadas, sendo as mais comuns em casos graves: síncope, angina, fadiga, dispneia e insuficiência cardíaca (GRIMARD BH, et al., 2016).

Diante da suspeita de estenose aórtica deve se proceder com a investigação. O padrão ouro para avaliação dessa condição é o ecocardiograma transtorácico com doppler. Nesse exame a estenose pode ser diagnosticada e classificada em leve, moderada ou grave. Na estenose aórtica leve tem-se pico da velocidade do jato aórtico 2 a 2,9 m/s, com gradiente médio menor que $20 \mathrm{mmHg}$. Já na moderada os parâmetros são o pico da velocidade do jato aórtico de 3 a $4 \mathrm{~m} / \mathrm{s}$, gradiente médio de 20 a $40 \mathrm{mmHg}$, área valvar de 1,0 a 1,5 $\mathrm{cm}^{2}$. Já na estenose aórtica grave, o pico da velocidade do jato aórtico é superior a $4 \mathrm{~m} / \mathrm{s}$, com gradiente médio maior que $40 \mathrm{mmHg}$, área valvar inferior a $1.0 \mathrm{~cm}^{2}$ (BORJA MM e OLVERA-ARREOLA SS, 2015; GRIMARD BH, et al., 2016).

Segundo o American Heart Association (AHA) além do ecocardiograma, deve ser realizado cateterismo cardíaco para avaliar a pervidade das coronárias para caso necessário realizar a revascularização do miocárdio na mesma intervenção cirúrgica. O cateterismo também deve ser realizado se houver discrepância entre a clínica e o ecocardiograma. O teste ergométrico também pode ser um exame complementar caso deseje avaliar a capacidade funcional do paciente ou prognóstico do pré-operatório (AHA, 2020).

Dessa forma, em decorrência do quadro de deterioração progressiva do ventrículo esquerdo, há a necessidade de intervenção médica, a fim de garantir a sobrevida do paciente. Morais LR, et al. (2021), demonstraram que a abordagem cirúrgica é a conduta mais adequada para casos de estenose aórtica importante e sintomática, sendo a única forma de resolução com eficiência a longo prazo capaz de devolver qualidade de vida, alívio dos sintomas e sobrevida ao paciente. Os autores afirmam, ainda, que mesmo para quadros assintomáticos, a correção cirúrgica pode ser indicada caso a fração de ejeção seja inferior a $50 \%$.

Os procedimentos cirúrgicos indicados para o tratamento da EA são a cirurgia convencional e o TAVI. O método convencional consiste na substituição da válvula aórtica estenosada por uma válvula biológica ou mecânica por meio da abertura da aorta, remoção da válvula acometida e posterior implantação da prótese valvar, a qual será suturada diretamente no coração. Logo, a possibilidade de operar o coração parado sob visão direta faz da cirurgia convencional um procedimento eficiente com incidência de vazamentos paravalvares quase nulos e necessidade de implantação de marcapasso em menos de $10 \%$ dos casos (AMARAL HLP, et al., 2021; BUZZATTI N, et al., 2020).

Para a realização dessa técnica, entretanto, é necessário que o paciente seja submetido a uma série de procedimentos que representam uma fonte de morbidade, como por exemplo, anestesia geral associada à intubação, abertura torácica, circulação extracorpórea e parada cardioplégica. Além disso, a abordagem cirúrgica convencional, traz associada a esses riscos, contraindicações tais como sexo feminino, doença arterial coronariana, idade avançada, cirurgia cardíaca prévia, emergência cirúrgica, disfunção ventricular, hipertensão pulmonar e múltiplas comorbidades. Dessa forma, cerca de um terço dos pacientes com essa patologia apresentam risco significativo para realização de intervenções cirúrgicas convencionais, ou seja, mais invasivas (MORAIS LR, et al., 2021).

Nestes casos o TAVI torna-se um forte candidato, uma vez que oferece uma abordagem menos invasiva e, portanto, de menor risco, ocupando assim o lugar de técnica padrão-ouro para pacientes de alto risco e uma opção para médio e baixo risco. Assim, o TAVI consiste na inserção de uma prótese biológica, montada em um stent, que vai ser introduzida na corrente sanguínea através de um cateter. Essa é uma técnica menos invasiva, que faz implantação da prótese sem suturas ou com suturas mínimas através de um acesso percutâneo sem necessidade de circulação extracorpórea e parada cardíaca (LEVETT J, et al., 2020; FOLLADOR W, et al., 2018).

Essas características tornaram o TAVI o procedimento de escolha para pacientes sintomáticos que possuem maior risco de complicações e mortalidade cirúrgicos e idosos acima de 75 anos, visto que a técnica garante áreas valvares maiores e com melhores desempenhos hemodinâmicos, além de reduzir o tempo cirúrgico e de recuperação pós-operatória. Além disso, ao contrário da cirurgia convencional, no TAVI a 
válvula nativa estenosada não é removida e sim esmagada pela prótese inserida pelo cateter, fazendo com que a nova válvula se fixe no local adequado devido à força radial de seu stent e não por suturas. Por isso, essa técnica faz com que haja um aumento no índice de vazamentos paravalvares e necessidade de marcapassos (BUZZATTI N, et al., 2020; PINTO BCM, et al., 2020).

O implante transcateter da válvula aórtica pode ser feito por diferentes sítios de acesso, dentre eles, o transapical e transfemoral. As principais diferenças entre eles é a necessidade de anestesia geral, acesso cirúrgico percutâneo e tempo intermediário de recuperação pós-operatória na técnica transapical, enquanto a transfemoral utiliza apenas anestesia local, acesso percutâneo e possui baixo tempo de recuperação pósoperatória (BUZZATTI N, et al., 2020). Apesar dos benefícios, pacientes com doença vascular periférica podem não ser elegíveis para a abordagem com o acesso transfemoral devido a taxas mais altas de dano vascular (WANG D, et al., 2020).

Ambas as técnicas (convencional e TAVI) possuem a mesma taxa de mortalidade dentro de um período de trinta dias, bem como riscos semelhantes de complicações como acidente vascular encefálico, infarto agudo do miocárdio e sangramentos. No entanto, na técnica de TAVI, observou-se uma superioridade em relação à complicações como a necessidade de marca-passo permanente, oclusão dos óstios coronarianos, ruptura anular, perfuração ventricular, regurgitação paravalvular, bloqueio cardíaco, posição errada ou embolização do dispositivo, acidente vascular cerebral, lesão vascular, dissecção toracoabdominal, injúria renal aguda e Leak paravalvar, quando em comparação com a cirurgia de reparo convencional (BORRACCI RA, et al., 2020; MORAIS LR, et al., 2021).

As pesquisas sobre o uso do TAVI em pacientes com baixo risco cirúrgico ainda estão em andamento. Já foram publicados benefícios para a implantação nestes pacientes, mas a durabilidade a longo prazo das válvulas bioprotéticas aórticas transcateter ainda é uma questão desconhecida (FAUVEL C, et al., 2020). Normalmente, os estudos sobre a durabilidade a longo prazo das válvulas biológicas avaliam pacientes com maior risco cirúrgico e, portanto, contraindicados à cirurgia convencional. Atrelado a isso, a grande maioria desses indivíduos possuía prognóstico desfavorável e idade avançada, o que determinou curtos períodos de acompanhamento após a intervenção (FAUVEL C, et al., 2020; RIBEIRO H, et al., 2018).

Dentre os pacientes que possuem maior risco perioperatório e piores resultados pós-cirúrgicos para o TAVI, destacam-se os portadores de EA com disfunção ventricular esquerda (DVE) associada ao baixo fluxo e ao baixo gradiente. Entretanto, um estudo realizado em 2019 observou que esses pacientes com maior risco, quando submetidos ao TAVI, tiveram grande melhora na fração de ejeção do ventrículo esquerdo (FEVE) e os resultados periprocedimentos e clínicos pós-TAVI são similares aos resultados obtidos pelos pacientes com DVE leve. Além disso, os pacientes com DVE grave apresentaram maior benefício relacionado com o aumento da FEVE quando comparado aos pacientes com DVE preservada. Sendo assim, a pesquisa concluiu que é indicado a realização do TAVI em pacientes graves independente do grau de disfunção ventricular esquerda e dos resultados da ecocardiografia sob estresse com dobutamina (MAES F, et al., 2019).

Em relação a implantação do TAVI em grandes centros, agências internacionais como a inglesa National Institute for Health and Care Excellence (NICE) e a canadense Canadian Agency for Drugs and Technologies in Health (CADTH), reconhecem os benefícios do TAVI em pacientes com estenose aórtica grave e recomendam o uso da técnica. No Brasil, a Comissão Nacional de Incorporação de Tecnologia (CONITEC) expõe empecilhos para a incorporação do procedimento no Sistema Único de Saúde (SUS), dentre eles a necessidade de especialização dos médicos responsáveis e estrutura hospitalar com suporte de sala hemodinâmica e cirurgia cardíaca, que não estão disponíveis em todos os centros brasileiros (BRASIL, 2021).

Além disso, devem ser considerados os desafios que pautam a indústria farmacêutica e biomédica, que necessitam de licenças para a autorização do uso de seus produtos em humanos, o que pode levar de meses a anos, a depender de quão rápido os estudos conseguem ser desenvolvido, o que é impactado por diversas variáveis, como o financiamento. Assim, mesmo com todas as evidências científicas, esses fatores apresentaram uma relação custo-utilidade desfavorável para o âmbito econômico do país e, atualmente, 
ultrapassam os limites de pagamento para implantação da tecnologia (BRASIL, 2021; PINTO BCM, et al., 2020).

\section{CONSIDERAÇÕES FINAIS}

Tendo em vista os fatos apresentados, reforça-se que o Implante Valvar Aórtico Transcateter oferece benefícios no tratamento da estenose valvar aórtica, é menos invasivo e apresenta menor risco quando comparado à cirurgia convencional, principalmente em pacientes graves ou considerados inoperáveis. No entanto, o custo, a falta de estrutura hospitalar, bem como de médicos especializados e de testes relacionados aos seus resultados a longo prazo impedem que tal procedimento ofereça efeitos mais favoráveis a um número maior de indivíduos. Isto posto, é fundamental o planejamento e investimento nos hospitais e equipes a fim de otimizar o acesso a esse tipo de procedimento. Ademais, deve-se incentivar o aperfeiçoamento dos profissionais, aprimorando as técnicas de implantação e realizando mais estudos acerca do pós-operatório em pacientes com EV leve ou moderada.

\section{REFERÊNCIAS}

1. AMARAL HLP, et al. Alternativas para substituição da valva aórtica em casos de estenose. Revista Eletrônica Acervo Científico, 2021;18: e5824.

2. AMERICAN HEART ASSOCIATION (AHA). Guideline for the Management of Patients With Valvular Heart Disease: A Report of the American College of Cardiology/American Heart Association Joint Committee on Clinical Practice Guidelines, 2020. Disponível em: https://www.ahajournals.org/doi/10.1161/CIR.0000000000000923 Acessado em 16 de junho de 2021.

3. BORJA MM, OLVERA-ARREOLA SS. Cuidado de enfermería a la persona con estenosis aórtica severa posterior al implante valvular aórtico transcatéter. Investigación en Enfermería: Imagen y Desarrollo, 2015; 17(1): 45-64.

4. BORRACCI RA, et al. Metaanálisis sobre los resultados a 30 días del implante valvular aórtico transcatéter en pacientes con riesgo intermedio en Argentina. Revista Argentina de Cardiología, 2020; 88(2):110-117.

5. BRASIL. Comissão Nacional de Incorporação de Tecnologia no SUS - Implante percutâneo de válvula aórtica (TAVI) para tratamento da estenose aórtica grave em pacientes inoperáveis: Relatório de recomendação. 2021. Disponível em:

http://conitec.gov.br/images/Consultas/Relatorios/2021/20210317_Relatorio_CP_15_TAVI_estenose_aortica_grave. pdf. Acessado em: 16 de junho de 2021.

6. BUZZATTI N, et al. Comparing traditional aortic valve surgery and transapical approach to transcatheter aortic valve implant. Eur Heart J Suppl., 2020; 22(Suppl E):E7-E12

7. FAUVEL C, et al. Durability of transcatheter aortic valve implantation: A translational review. Archives of Cardiovascular Diseases, 2020; 113: 209-221.

8. FOLLADOR W, et al. Estenose valvular aórtica e o uso de TAVI: revisão narrativa das evidências publicadas e avaliação básica de custos. Jornal Brasileiro de Economia da Saúde, 2018;10(1): 36-44.

9. GRIMARD BH, et al. Aortic Stenosis: Diagnosis and Treatment. American Academy of Family Physicians, 2016; 1;93(5):371-378.

10. JOSEPH J, et al. Aortic Stenosis: Pathophysiology, Diagnosis and Therapy. The American Journal of Medicine, 130(3), 253-263.

11. KANWAR A, et al. T. Management of patients with aortic valve stenosis. Mayo Clinic Proceedings, 2018; 93(4):488508.

12. KUNTJORO I, et al. Cost-Effectiveness of Transcatheter Aortic Valve Implantation in Intermediate and Low Risk Severe Aortic Stenosis Patients in Singapore. Annals of the Academy of Medicine of Singapore, 2020;49(7):423-433.

13. LEVETT J, et al. Meta-Analysis of Transcatheter Versus Surgical Aortic Valve Replacement in Low Surgical Risk Patients. The American Journal of Cardiology, 2020; 15;125(8):1230-1238.

14. LIU Z et al. Transcatheter aortic valve implantation for aortic stenosis in high surgical risk patients: A systematic review and meta-analysis. PLoS One, 2018;13(5):e0196877.

15. MAES F, et al. Outcomes From Transcatheter Aortic Valve Replacement in Patients With Low-Flow, Low-Gradient Aortic Stenosis and Left Ventricular Ejection Fraction Less Than 30\%: A Substudy From the TOPAS-TAVI Registry. JAMA Cardiol., 2019; 1;4(1):64-70.

16. MANGIONE FM, et al. Principais estudos clínicos sobre implante de válvula aórtica transcateter. Revista da Sociedade de Cardiologia do Estado de São Paulo, 2017; 27(1): 20-5.

17. MORAIS LR, et al. O implante de valva aórtica transcateter no tratamento da estenose aórtica: perspectivas e desafios. Brazilian Journal of Health Review, 2021; 2(4): 4051-4065.

18. OTTO CM, et al. ACC Expert Consensus Decision Pathway for Transcatheter Aortic Valve Replacement in the Management of Adults With Aortic Stenosis: A Report of the American College of Cardiology Task Force on Clinical Expert Consensus Documents. Journals of the American College of Cardiology, 2017; 69(10): 1313-1346. 
19. PINTO BCM, et al. Troca valvar endovascular: vantagens para pacientes de risco intermediário e alto em relação ao tratamento convencional. Revista Eletrônica Acervo Saúde, 2020;12(10):e4669.

20. PIO G, et al. A Evolução do Implante Valvar Aórtico Transcateter: Revisão de Literatura. Brazilian Journal of Health Review, 2021, 4: 8788-8800.

21. PONTES J, et al Experiência inicial e pioneira do implante de valva aórtica transcateter por via femoral ou ilíaca. Revista Brasileira de Cirurgia Cardiovascular, 2013, 28(2): 208-16.

22. RIBEIRO H, et al. Transcatheter Aortic Valve Replacement in Patients With Low-Flow, Low-Gradient Aortic Stenosis. Journal Of The American College Of Cardiology, 2018; 71(12): 1297-1308.

23. WANG D, et al. Transcatheter aortic valve implantation versus surgical aortic valve replacement for treatment of severe aortic stenosis: comparison of results from randomized controlled trials and real-world data. Brazilian Journal of Cardiovascular Surgery, 2020; 35(3): 346-367.

24. ZHENG KH et al. Pathophysiology of Aortic Stenosis and Future Perspectives for Medical Therapy. Cardiology Clin, 2020; 38(1): 1-12. 